\title{
The interaction between skill, postures, forces and back pain in wool handling
}

\author{
Stephan Milosavljevic ${ }^{1}$, Diane E Gregory ${ }^{2}$, Poonam Pal ${ }^{1}$, Allan B Carman ${ }^{1}$, Peter D \\ Milburn $^{3}$, Jack P Callaghan $^{4}$ \\ ${ }^{1}$ Centre for Physiotherapy Research, School of Physiotherapy, University of Otago, Dunedin, \\ New Zealand \\ ${ }^{2}$ Department of Orthopedic Surgery, University of California, 200 W. Arbor Drive 8894, San \\ Diego, California, USA \\ ${ }^{3}$ School of Physiotherapy and Exercise Science, Gold Coast Campus, Griffith University, \\ Queensland, Australia \\ ${ }^{4}$ Faculty of Applied Health Sciences, Department of Kinesiology, University of Waterloo, \\ Waterloo, Ontario, Canada
}

\section{List of authors}

Stephan Milosavljevic (corresponding author), Centre for Physiotherapy Research, School of Physiotherapy, University of Otago,PO Box 56, Dunedin, New Zealand, 64-3-479-7193, Fax: 64-3-479-8414, stephan.milosavljevic@otago.ac.nz;

Diane E Gregory, Department of Orthopedic Surgery, University of California, $200 \mathrm{~W}$. Arbor Drive 8894, San Diego, California, USA,1-858-822-0148, degregory@ucsd.edu;

Poonam Pal, Centre for Physiotherapy Research, School of Physiotherapy, University of Otago, PO Box 56 Dunedin, New Zealand, 64-3-479-7460, Fax: 64-3479-8414, poonam.pal@otago.ac.nz;

Allan B Carman, Centre for Physiotherapy Research, School of Physiotherapy, University of Otago, PO Box 56 Dunedin, New Zealand, 64-3-479-3485, Fax: 64-3479-8414, allan.carman@otago.ac.nz;

Peter Milburn, School of Physiotherapy and Exercise Science, Gold Coast Campus, Griffith University, QLD 4222, Australia, p.milburn@griffith.edu.au;

Jack P Callaghan, Faculty of Applied Health Sciences, Department of Kinesiology, University of Waterloo, Waterloo, Ontario, Canada, 1-519-888-4567 (ext 37080), Fax: 1-519-885-0470, callagha@healthy.uwaterloo.ca

\section{Current word count $=\mathbf{2 9 9 6}$ words}




\begin{abstract}
Wool handling is an important rural occupation where workers process 200 or more fleeces daily, separating them into various quality components. Loads and postures they experience carry substantial risk of low back pain (LBP). Although a formal skill training structure exists, interaction with loads and LBP is unknown. We examined whether skill and LBP influenced trunk postures and loads of 60 wool handlers representing 3 skill levels. LBP prevalence ranged from $20 \%$ for junior (lowest skill) to $45 \%$ for open class (highest skill) wool handlers. Open class wool handlers demonstrated increased lateral bend and more axially twisted postures, generating greater medio-lateral shear forces and lateral bend and axial twist moments. LBP was associated with open class wool handlers spending more time in severe axially twisted postures. These findings suggest that skill-based training needs to be reviewed to reduce the quantity of axially twisted posture which may help reduce the prevalence of LBP in this workforce.
\end{abstract}

\title{
Keywords
}

Wool handling, work postures, skill, low back pain, cumulative loading. 


\section{Introduction}

Wool is an important rural commodity manually harvested by shearers, sorted by wool handlers, pressed into 180 to $200 \mathrm{~kg}$ bales for market purchase, and ultimately processed into yarn, cloth and other tertiary products. Recent investigation of skill and low back pain (LBP) in shearers demonstrates that skill can discriminate for differences in working posture and lifetime prevalence of occupational LBP. Shearers with a history of LBP also demonstrated significantly greater left medio-lateral and axial twist moments during their work tasks. Recommendations were made for industry training organisations to focus on reducing positional asymmetry from the flexed working postures of shearers.

There has been very little research on the injury risk or biomechanical, ergonomic or physiological demands of wool handlers (Gregory et al. 2009). Workplace observation (Figure 1) reveals a predominantly female workforce working in a variety of repetitive postures (often stooped) picking up in excess of 200 fleeces per day, each weighing between 5 to $10 \mathrm{~kg}$, and "throwing" these onto wool sorting tables where they are prepared, graded, and separated into various quality components. Gregory et al (2009) has recently investigated shoulder postures and loads of wool handlers and observed that highest skill (open class) spends significantly more time in neutral or mild shoulder postures. They suggest that risk of sustaining injury to the upper limb of the open class wool handler may be less than that of other skill levels and argue the potential importance of this observation for industry workforce training. However, no examination has been made of trunk postures and associated loads on the spine. 
The aim of this study is to investigate the spinal loads and trunk postures of wool handling and to determine whether skill and/or LBP have any influence on these variables. 


\section{Method}

\subsection{Participants}

Following ethical approval from the University of Otago Human Ethics Committee, 60 wool handlers (20 junior, 20 senior, and 20 open class) were recruited from two major wool harvesting competitions (Shearing Sports Inc. 2008) held in the South Island of New Zealand. Participant recruitment involved accessing competition schedules constructed for each skill level on the day of competition, and approaching wool handlers prior to competition until recruitment of 20 consenting wool handlers for each skill classification was completed. Following consent, participating wool handlers were surveyed for age (years), work experience (years), height (m), weight $(\mathrm{kg})$, and the number of previous episodes of occupational LBP requiring clinical intervention (LBP-Clin) during their working lifetime.

During these competitions each wool handler's competitive score is formally judged on both the time taken to process the fleece and the quality of the processed fleece (Shearing Sports Inc. 2008, TECTRA NZ 2008). The wool handlers were recruited during the peak summer shearing season where there are considerable numbers of resident and itinerant workers harvesting wool and competing in regional wool handling competitions for peer and potential upward revision of skill. As this is common practice for most of the work force the participants in this study were accepted as a valid representation of wool handlers in New Zealand. The tasks undertaken during the competition were accepted as being representative of actual workplace conditions and while the emphasis is on two main parameters: quality of the sorted wool and the time taken to process and sort the wool this study only sued the temporal and postural properties of the required tasks for analysis, Each wool handler was registered with Searing Sports administration (Shearing Sports Inc, 2008; TECTRA NZ, 2008) and their previously judged ranking (junior, senior \& open 
class - based on the above parameters) used as the entry criteria for competition at that skill level.

Each wool handler was videotaped while processing three fleeces during their competitive heats. Their tasks involved gathering, preparing, grading, and separating the various wool components of the fleece - in a manner similar to that required in their working environment. They were videotaped from two different camera locations allowing full observation and recording of the wool handling tasks (Sutherland et al. 2007, Pal et al. 2010).

\subsection{Video capture}

Each videotape was captured to AVI digital format, with each wool handler's video file of 3 fleeces being sorted ranged from 4.5 to 9.0 minutes in duration dependent on level of skill. Each captured video trial was then analyzed using 3DMatch (University of Waterloo, Waterloo, Ontario, Canada) at the accepted sampling rate of 3 frames/sec (Andrews and Callaghan, 2003).

\subsection{Cumulative posture and force analysis}

Cumulative moments about $L 4 / 5$ were calculated by selecting previously validated sagittal, coronal and axial trunk posture bins (Sutherland et al, 2008) during each frame of the video, and included external 3D hand and upper limb forces, allowing the calculation of 3D moments about $L 4 / 5$ joint. For a more detailed description of 3DMatch, refer to Gregory et al (2006). Each full fleece was accepted as 55.0N of load with $5.6 \mathrm{~kg}$ being the average accepted weight of a cross bred fleece in New Zealand (Rowland 2006). Minor trimmings of wool undertaken during the skirting process were accepted as creating no more than $1.0 \mathrm{~N}$ load, while wool containment baskets were accepted as a $10.0 \mathrm{~N}$ force and the "belly" wool removed during the initial part of shearing generating no more than 5.0N. The moments calculated for 
each frame were integrated over the entire duration of sorting the 3 fleeces in order to determine cumulative loads, which could then be extrapolated to an 8-hour working day. No account was taken of the inertial loads experienced during handling

\subsection{Statistical analysis}

All analyses were undertaken wit hthe use of SPSS ${ }^{\mathrm{TM}}(\mathrm{V}$ 16.0) statistical software. Skill was categorically classified $(0=$ junior; $1=$ senior; $2=$ open $)$, as was history of LBP-Clin $(0=$ No; 1 = Yes $)$. All other explanatory variables - personal, anthropometric, and work experience - were recorded as continuous data. The response variables were cumulative loads and postural data. The personal, anthropometric, and work related variables are summarised by skill level in Table 1, and explored for statistically significant differences between skill levels and LBP-Clin categories, and the postural and cumulative loading data are presented in Table 2. The influence of both skill and previous LBP-Clin on the percentage of cumulative time spent in each of 10 working postures and on each of 14 cumulative loads was explored firstly with a bivariate linear regression model. In order to optimally identify the influence of each explanatory variable the bivariate acceptance threshold into the multivariate model was contingent upon $\mathrm{p} \leq 0.20$ (Altmanm 1996, pp. 349). As BMI, age and experience were also considered as potential confounders they were included in the multivartiate analysis of each independent variable. The multivariate linear model used a dichotomous indicator variable (dummy variable) to represent each level of skill, and a dichotomous history of LBP-Clin (yes/no) as categorical explanatory variables.. As wool handlers formally improve their productivity (tally) by undertaking skill training programmes it was considered that skill was the more important explanatory variable for inclusion in the stepwise model. The use of a stepwise model allowed us to focus on the effects of skill and LBP-Clin and their interaction as predictors of spinal loading and posture while spinal loading and posture were controlled for the inclusion of selected covariates as potential 
confounding variables (Altman, 1966, pp. 325-326 \& 340-351). This also allowed direct comparison with recently published shearing research that also explored the effects of skill and LBP-Clin. (Pal et al , 2010).The statistically significant results for these multivariate analyses are presented in Tables 3 and 4. For each model, each coefficient represents the difference between the mean of the group, represented by the particular term in the model and the mean of the referent group consisting of the aggregate of all groups not differentiated by the model. 


\section{Results}

\subsection{Anthropometric and work characteristics}

The combined sample of 60 wool handlers had a mean age of 27.6 years, mean weight of $72.0 \mathrm{~kg}$, mean height of $1.65 \mathrm{~m}$, and mean BMl of $26.3 \mathrm{~kg} / \mathrm{m}^{2}$ across all skill levels (Table 1). Fifty four (90\%) of the sample were female and the 6 males were distribute across the 3 skill classifications (open $=3$, senior $=2$, junior $=1$ ). Mean work experience was 9.4 years with a mean time-predicted daily tally of 231.5 fleeces. Twenty four (40\%) of the wool handlers described having at least one previous episode of LBP-Clin. Although there were no significant associations for age, experience, skill, weight, BMI, with LBP-Clin; increased age $(p=0.001)$, height $(p=0.004)$, work experience $(p<0.001)$ and tally $(p<0.001)$ were significantly associated with the highest (open class) skill level.

Insert Table 1 about here

\subsection{Mean group values for posture and cumulative load.}

When aggregated across groups (Table 2) the predominant percentage of time (63.9\%) was spent in mild flexion (from $25.0^{\circ}$ to $45.0^{\circ}$ ), neutral lateral bend $(79.9 \%)$, and neutral axial twist (73.5\%). Mean cumulative compressive force and force weighted cumulative compression about L4/5 (Parkinson and Callaghan, 2007) were 48.7MN.s and 48.9MN.s respectively (Table 2). Cumulative joint anterior and reaction anterior shear were 2.5MN.s and 5.7MN.s with right and left medial to lateral shear of 0.5MN.s and 0.6MN.s. Cumulative joint posterior shear and reaction posterior shear were $47.7 \mathrm{kN} . \mathrm{s}$ and $10.1 \mathrm{kN}$.s respectively. Cumulative extensor moment was 2.1MNm.s, cumulative flexor moment $2.4 \mathrm{kNm} . \mathrm{s}$, cumulative right and left lateral bend moments were $0.2 \mathrm{MNm}$.s and $0.2 \mathrm{MNm}$.s while cumulative right and left axial twist moments were $26.6 \mathrm{kNm} . \mathrm{s}$ and $28.5 \mathrm{kNm} . \mathrm{s}$. 
Insert Table 2 about here

\subsection{Effect of skill and LBP-Clin on cumulative percent time in working postures}

Senior class wool handlers (Table 3) worked for a mean $5.4 \%$ less in mild flexion $(p=$ 0.029) when compared to the open and junior class. Open class spent $5.1 \%$ less time $(p=0.004)$ in neutral lateral bend and $4.0 \%$ more time $(p=0.005)$ in mild lateral bend, while senior class spent $10.9 \%$ more time in neutral and $10.2 \%$ less time in mild axial twist. Open class with a history of LBP-Clin spent $1.5 \%$ more working time in severe axial twist. These five models explain $8 \%, 14 \%, 13 \%, 10 \%, 9 \%$ and $31 \%$ of data variance for mild flexion, neutral lateral and mild lateral bend, and neutral, mild and severe axial twist respectively (Table 3).

Insert Table 3 about here

\subsection{Effect of skill and LBP-Clin on cumulative load}

Neither skill nor a previous history of LBP-Clin, nor the interaction between these two variables, had any statistically significant influences on either compressive or anterior/posterior shear cumulative forces. However the open class demonstrated significantly greater right and left medio-lateral reaction shear, right and left lateral bend moments, and right and left axial twist moments (Table 4) than the senior and Junior wool handlers. The models for these six variables respectively explain $14 \%$, $8 \%, 10 \%, 15 \%, 17 \%$ and $14 \%$ of data variance. BMI, age and experience were also included in each of the multivariate load models but had no significant influence on the strength of these models. 


\section{Discussion}

The primary aim of this study was to explore how skill and a history of LBP-Clin affect cumulative trunk postures and low back loads of wool handlers. Descriptively there was a 9.1 year mean difference in age and a 4.5 year mean difference in work experience between junior and open class wool handlers. Despite a significant height difference for open class there were no differences in weight or BMI amongst the three skill levels, indicating little discriminative influence for these anthropometric variables. Consistent with industry advice (Rowland, 2006) and workplace observation, $90 \%(n=54)$ of this sampled workforce were female and thus the small number of males $(n=6)$ distributed across the 3 skill levels could not provide any numerical strength to allow any comparative calculation of posture and load differences between males and females. Skill progression from junior to senior wool handler did not demonstrate any difference in estimated tally (fleeces handled per day) indicating the progression from junior to senior wool handler is predominantly based on quality score of the prepared fleece as judged by their peers. The progression to open class increased tally by an average of only 15 fleeces per day and suggests that quality fleece preparation is the paramount issue during wool handling tasks at least in the competition setting. Although there were observable differences in working lifetime prevalence of LBP-Clin particularly between junior (20.0\%) and senior wool handler (50.0\%) the progression to open class (45\%) did not increase prevalence and indicates that exposure to risk factors is likely to be lower than that of progression to an open class (76\%) shearer (Pal et al. 2010).

When compared to the working postures of shearers (Pal et al. 2010) wool handlers spend noticeably less cumulative time in severe trunk flexion, and more time in mild to severe lateral bend, and mild to severe axial twist. These differences directly influence the magnitude of cumulative loads at $L 4 / 5$ where wool handler compression (50.0MN.s v 81.9MN.s) and anterior shear (2.4MN.s v 5.3MN.s) forces are also 
noticeably less, reflecting the greater time spent by wool handlers in standing and less severe stooped postures. Not surprisingly the percentage of time that wool handlers work in a neutral to an extended posture generates minor but measurable posterior shear forces (47.7kN.s) and flexor moments $(2.4 \mathrm{kNm} . \mathrm{s})$. The wool handlers' cumulative left $(0.6 \mathrm{MN} . \mathrm{s} \vee 33.72 \mathrm{kN} . \mathrm{s})$ and right $(0.5 \mathrm{MN} . \mathrm{s} \vee 16.4 \mathrm{kN} . \mathrm{s})$ medio-lateral shear forces, left (0.2MNm.s v 8.6kNM.s) and right (0.2MNm.s $v 16.2 \mathrm{kNM} . \mathrm{s})$ lateral bend moments, and left (28.5kNm.s $\vee 3.4 \mathrm{kNM} . \mathrm{s})$ and right $(28.6 \mathrm{kNm} . \mathrm{s} \vee 11.3 \mathrm{kNM} . \mathrm{s})$ axial twist moments are substantially greater than the shearers' (Pal et al. 2010) reflecting the much greater percentage of time wool handlers work in asymmetric yet less flexed postures.

A multivariate exploration of the effects of skill and previous LBP-Clin on wool handlers showed that senior class wool handlers spent significantly less time in mild flexion, significantly more time in neutral and significantly less time in mild axial twist. Open class wool handlers spent less time than senior and junior wool handlers in neutral and more time in mild lateral bend. While statistically significant, the percentage of variance explained by these models (from $8.0 \%$ to $14.0 \%$ ) is not strong. Although the multivariate model did not accept LBP-Clin as a predictor for these postures open class wool handlers with a history of LBP-Clin spent significantly more time in severe axial twist postures in comparison to the referent group (all other wool handlers). Although this is only a $1.5 \%$ increase in time this interaction variable explains $31 \%$ of the variance for percentage time in severe axial twist and is a statistically strong $(p<0.0001)$ result. Post hoc analysis shows that $1.5 \%$ of an 8 hour work day is 7.2 minutes extra time spent in severe axial twist. This amount of increased time exposure in severe axial twist undertaken in various combinations of lateral bend and trunk flexion would be a plausible mechanism for creating increased risk of LBP in this workforce. While open class wool handlers generated significantly greater cumulative right and left reaction shear, right and left lateral bend and axial 
twist moments about L4/5 the addition of LBP-Clin or its interaction with the three skill levels did not contribute any further statistical strength to the models. The contribution of the open class skill to the variance for these load models was not strong ranging from $8.0 \%$ to $17.0 \%$ (Table 4 ).

It is interesting to observe that bivariate analysis showed no relationship between skill and LBP-Clin and any of age, experience, height, weight and BMI. This is different from the observed significant relationship between skill and LBP in shearers (Pal et al. 2010). Although the medio-lateral and axial twist postures and loads were significantly greater for the wool handlers compared to the shearers the shearers had a substantially greater cumulative compressive and anterior shear force. Post hoc analysis also demonstrates a wool handler mean compressive force of $1691 \mathrm{~N}$ and a $21 \mathrm{~N}$ joint anterior shear force about $L 4 / 5$, while for the shearers these were $2844 \mathrm{~N}$ and $185 \mathrm{~N}$ respectively. Thus the mean compressive and anterior shear forces for the shearers are 1.7 and 8.8 greater than that of the wool handlers and mean shearer compressive forces are within $83 \%$ of the $\mathrm{NIOSH}$ action limit of 3433N (Milosavljevic et al. 2004, Gregory et al. 2006).

These lesser cumulative loads and more variable postures for wool handlers are more likely to be within the safety tolerances of loading for the spine and are probably the main reason for the lower prevalence rate for open class wool handlers (45\%) compared to open class shearers (76\%) whose work generates greater spinal loads in more extreme flexed postures (Pal et al. 2010). Although these wool handler cumulative compressive loads are less than the shearers, it is important to note that they are still considerably greater than those described by Kumar (1990) and Norman et al (1998) for at risk health care (15MN.s) and auto assembly workers (20MN.s) respectively (Gregory et al. 2006). While shearers appear to carry high risk for LBPClin that is associated with sustained posture, substantial cumulative loads, 
increased medio-lateral shear forces and lateral bend and axial twist moments, pen class wool handlers carry risk for LBP-Clin that is more associated with excessive axial twist postures. Although the open class wool handlers had greater magnitudes for all asymmetric loads, these were not associated with LBP-Clin. It may also be that the numbers per skill classification were insufficient to discriminate any further relationships for the LBP-Clin variable.

Ann absence of LBP-Clin at least in open class wool handlers is associated with reduced amount of time in severe axial twist, so a focus on wool handler training to reduce the magnitude and time in axially twisted postures might be an important intervention. Although the results were not significant, attention to reducing lateral bend postures would also be a logical step since such postures will contribute to the observed differences in lateral bend moments and shear forces. Ways to reduce these extremes of axially twisted posture through wool handling instruction will ultimately determine the effect of such interventions on risk of LBP-Clin in this workforce.

This research has focused on how current skill ranking and a history of LBP-Clin are associated with observed trunk postures and spinl loads in a stepwise regression model. This cross-sectional design cannot determine causal links between postures and loads and the prevalence of LBP-Clin. In essence, this is a case-control study where those with a history of LBP-Clin demonstrate more extreme working postures and loads compared to those who have no such history. Although it appears logical that extremes of load and posture are predictors of LBP-Clin, it is also plausible (but considerably unlikely) that those who have had previous LBP-Clin have adapted to more extreme axially twisted working postures as a consequence of prior injury.. Although 60 participants took part, sub-classification by three levels of skill weakens the argument for strength of association between skill, posture and load. Similarly the 
interaction between skill and LBP-Clin will also have a weaker argument for any observable effects and thus this study does not have the numerical strength for a robust and full examination of these associations. This cumulative postural and load analysis is based on a 4.5 - 9.0 min sample (dependent on skill level) and the results extrapolated to an $8 \mathrm{~h}$ working day. Thus the effects of fatigue and repetition on load and postural demands during a demanding set of work tasks have not been accounted for in this analysis. Hence external validity of this research to the standard working day of the wool handling workforce is unclear and will require a larger and powered sample in a longitudinal design using similar postural analysis. . Such research would help to validate the current results.

Sample size and the multivariate limitations of 24 postural and load response variables as well as 11 anthropometric and work-place explanatory variables mean that some of these significant results may be a chance occurrence. Given the multivariate stepwise testing of a limited number of variables that meet the $p<0.20$ entry criteria some of the results may also be over-optimistic with regards to the strength of the model and its importance. In hindsight a larger sample of at least $n=$ 120 would have satisfied recommended criteria $(n / 10)$ for the number of explanatory variables to fit a multivariate model. Given the sample size limitations ( $n=60)$ we focussed on stepwise model that explored how skill and LBP-Clin were associated with trunk posture and spinal load and chose bivariate models to pre-select covariates. While this successfully limited the number of explanatory variables in each model it is acknowledged that some of the significant observations may be over-optimistic (Altman, 1966 pp. 349-351).

\section{Conclusions}

Open class wool handlers spend more time in asymmetric lateral bend and axial twist postures and logically generate greater medio-lateral forces and lateral bend and 
axial twist moments in these postures. Dependent on skill level the working lifetime prevalence of LBP-Clin ranged from 25 (junior class) to 44\% (open class), considerably less than the $76 \%$ working lifetime prevalence for LBP-Clin for open class shearers. Open class wool handlers with a history of LBP-Clin work for longer periods of time in severe axial twist postures which may be the factor that places them at increased risk of low back injury. Although the cumulative compressive and shear forces (48.7MN.s and 2.5MN.s respectively) are substantially less than that generated by shearers, the cumulative compressive forces are still considerably greater than that described for other at-risk populations. While the results of this study cannot clearly associate an increasing prevalence of LBP-Clin with increasing skill it may be that the magnitude of these compressive and shear forces are still a component of low back injury in this workforce. These results suggest a focus on reducing the magnitude and duration of axially twisted work postures in the wool handling workforce will help reduce the risk and prevalence of occupational LBP.

\section{Acknowledgments}

The authors acknowledge the funding support from:

Vernon Willey Trust; and the New Zealand Health Research Council Professor Callaghan is also supported by a Canada Research Chair (CRC) in Spine Biomechanics and Injury Prevention. 


\section{References}

Altman, D.G., 1999. Practical Statistics for Medical Research. Chapman \& Hall. London. .

Andrews, D.M., Callaghan, J.P., 2003. Determining the minimum sampling rate needed to accurately quantify cumulative spine loading from digitised video. Appl Ergon, 34 (6), 589-595.

Gregory, D.E., Pal, P., Carman, A.B., Milosavljevic, S., Callaghan, J.P., 2009. The effect of skill on shoulder postures and moments of force in the wool harvesting industry. Int J Occup Saf Ergon, 15(4),409-418.

Gregory, D.E., Milosavljevic, S., Callaghan, J.P., 2006. Quantifying low back peak and cumulative loads in open and senior shearers in New Zealand: Examining the effects of a trunk harness. Ergonomics, 49 (10), 968-981.

Kumar, S.,1990. Cumulative load as a risk factor for back pain. Spine, 15, 13111316.

Milosavljevic, S., Carman, A.B., Milburn, P.D., Wilson, B.D., Davidson, P.L., 2004.The influence of a back support harness on spinal forces during sheep shearing. Ergonomics, 47 (11), 1208-1225.

Norman, R., Wells, R., Neumann, P., Frank, J., Shannon, H., Kerr, M., 1998 A comparison of peak vs cumulative physical work exposure risk factors for the reporting of low back pain in the automotive industry. Clin Biomech, 13, 561573. 
Pal, P., Milosavljevic, S., Gregory, D.E., Carman, A.B., Callaghan, J.P., 2010 The influence of skill and low back pain on trunk postures and low back loads of sheep shearers. Ergonomics, 53(1), 65-73.

Pal, P., Gregory, D.E., Milosavljevic, S., Carman, A.B., Callaghan, J.P., 2008. A review of risk factors associated with low back injury in wool harvesting. $J$ Occup Health Saf, Aust NZ , 24(5), 435-453.

Parkinson, R. J., Callaghan, J. P., 2007. The role of load magnitude as a modifier of the cumulative load tolerance of porcine cervical spinal units: Progress towards a force weighting approach. Theor Iss Ergon Sci, 8(3), 185-200.

Rowland, G., 2006. Manager. Wool Harvesting Training. TECTRA NZ Ltd.

Shearing Sports NZ Inc. (2008). Description of NZ shearing competitions available at. http://www.shearingsports.co.nz/

Sutherland, C.A., Albert, W.J., Wrigley, A.T., Callaghan, J.P., 2007. The effect of camera viewing angle on posture assessment repeatability and cumulative spinal loading. Ergonomics, 50 (6), 877-889.

TECTRA NZ, 2008. Wool harvester training programme description available at http://www.tectra.co.nz/index.tpl 


\section{List of Figures}

Figure 1. Various tasks associated with wool handling. $A=$ Gathering wool from the shearing station. $B=$ Sorting wool on the floor. $C=$ Throwing an $\sim 5.5 \mathrm{~kg}$ fleece on to the wool sorting table. Note the variety of upright to stooped postures of the wool handlers compared to the fully stooped postures of the shearers.

\section{$\underline{\text { List of Tables }}$}

Table 1 Summary of anthropometric and work characteristics of wool handlers by skill level

Table 2 Summary percentage of time spent in various postures and the cumulative loads for each wool handler skill level

Table 3 Stepwise multivariate analyses for both wool handler skill level and LBPClin on cumulative percent time in working postures at 95\% CL

Table 4 Stepwise multivariate analyses for both wool handler skill level and LBPClin on cumulative loads at $95 \% \mathrm{CL}$ 
Table 1 - Summary of anthropometric and work characteristics of wool handlers by skill level

\begin{tabular}{|c|c|c|c|c|c|c|c|c|}
\hline \multirow[t]{2}{*}{ Characteristic } & \multicolumn{2}{|c|}{$\begin{array}{l}\text { Junior } \\
(n=20)\end{array}$} & \multicolumn{2}{|c|}{$\begin{array}{l}\text { Senior } \\
(n=20)\end{array}$} & \multicolumn{2}{|c|}{$\begin{array}{c}\text { Open } \\
(n=20)\end{array}$} & \multicolumn{2}{|c|}{$\begin{array}{c}\text { Total } \\
(n=60)\end{array}$} \\
\hline & Mean & SD & Mean & SD & Mean & SD & Mean & SD \\
\hline Mean age (yrs) & 22.9 & 5.7 & 27.9 & 7.6 & 32.0 & 10.0 & 27.6 & 8.7 \\
\hline Mean height (m) & 1.62 & 0.06 & 1.62 & 0.06 & 1.68 & 0.08 & 1.65 & 0.07 \\
\hline Mean mass (kg) & 69.8 & 14.9 & 69.8 & 14.9 & 73.1 & 14.3 & 72.0 & 16.3 \\
\hline Mean BMI (kg/m²) & 26.6 & 5.6 & 26.6 & 5.6 & 25.8 & 4.6 & 26.3 & 5.3 \\
\hline Mean work experience (yrs) & 4.9 & 5.1 & 9.3 & 6.0 & 14.2 & 8.4 & 9.4 & 7.6 \\
\hline Time predicted tally (fleeces) & 218.5 & 23.1 & 216.9 & 14.6 & 259.0 & 13.9 & 231.5 & 26.2 \\
\hline LBP-Clin (\%) & \multicolumn{2}{|c|}{$\begin{array}{c}25 \\
(n=5)\end{array}$} & \multicolumn{2}{|c|}{$\begin{array}{c}50 \\
(n=10)\end{array}$} & \multicolumn{2}{|c|}{$\begin{array}{c}45 \\
(n=9)\end{array}$} & \multicolumn{2}{|c|}{$\begin{array}{c}40 \\
(n=24)\end{array}$} \\
\hline
\end{tabular}


Table 2 - Summary percentage of time spent in various postures and the cumulative loads for each wool handler skill level

\begin{tabular}{|c|c|c|c|c|c|c|c|c|}
\hline \multirow{3}{*}{ Posture } & \multicolumn{2}{|c|}{$\begin{array}{l}\text { Junior } \\
(n=20)\end{array}$} & \multicolumn{2}{|c|}{$\begin{array}{l}\text { Senior } \\
(n=20)\end{array}$} & \multicolumn{2}{|c|}{$\begin{array}{c}\text { Open } \\
(n=20)\end{array}$} & \multicolumn{2}{|c|}{$\begin{array}{c}\text { Total } \\
(n=60)\end{array}$} \\
\hline & Mean & SD & Mean & SD & Mean & SD & Mean & SD \\
\hline & \multicolumn{8}{|c|}{ Percentage of time in posture } \\
\hline Extension $\left(>15^{\circ}\right)$ & 0.2 & 0.3 & 0.2 & 0.4 & 0.1 & 0.2 & 0.2 & 0.3 \\
\hline Neutral flexion $\left(<25^{\circ}\right)$ & 8.4 & 8.4 & 10.3 & 6.6 & 7.2 & 3.7 & 8.6 & 6.6 \\
\hline Mild flexion $\left(25-45^{\circ}\right)$ & 64.1 & 6.9 & 60.4 & 8.3 & 67.4 & 10.5 & 63.9 & 9.0 \\
\hline Severe flexion $\left(>45^{\circ}\right)$ & 27.3 & 6.0 & 29.1 & 8.0 & 25.2 & 9.5 & 27.2 & 8.0 \\
\hline Neutral lat bend $\left(<15^{\circ}\right)$ & 76.5 & 6.0 & 80.6 & 5.7 & 82.7 & 6.7 & 79.9 & 6.5 \\
\hline Mild lat bend $\left(15-30^{\circ}\right)$ & 19.8 & 4.9 & 16.9 & 4.4 & 14.8 & 5.3 & 17.2 & 5.2 \\
\hline Severe lat bend $\left(>30^{\circ}\right)$ & 3.7 & 1.6 & 2.6 & 2.3 & 2.5 & 2.3 & 2.9 & 2.1 \\
\hline Neutral ax twist $\left(<15^{\circ}\right)$ & 68.7 & 13.1 & 80.8 & 17.7 & 70.9 & 17.5 & 73.5 & 16.8 \\
\hline Mild ax twist $\left(15-30^{\circ}\right)$ & 29.7 & 12.3 & 18.7 & 17.4 & 28.2 & 17.2 & 25.6 & 16.3 \\
\hline Severe ax twist $\left(>30^{\circ}\right)$ & 1.6 & 1.1 & 0.5 & 0.5 & 0.9 & 0.9 & 1.0 & 1.0 \\
\hline Type of Load & \multicolumn{8}{|c|}{ Cumulative forces } \\
\hline Compression (MN.s) & 50.0 & 9.7 & 50.1 & 10.6 & 45.9 & 5.6 & 48.7 & 9.0 \\
\hline Weighted compression (MN.s) & 50.4 & 10.6 & 50.2 & 10.9 & 45.9 & 5.7 & 48.9 & 9.4 \\
\hline Joint anterior shear (MN.s) & 2.5 & 0.6 & 2.6 & 0.7 & 2.5 & 0.4 & 2.5 & 0.6 \\
\hline Reaction anterior shear (MN.s) & 5.7 & 1.4 & 5.9 & 1.7 & 5.4 & 0.9 & 5.7 & 1.4 \\
\hline Joint posterior shear (kN.s) & 50.2 & 45.2 & 58.4 & 34.8 & 34.6 & 18.6 & 47.7 & 35.5 \\
\hline Reaction posterior shear (kN.s) & 12.0 & 10.0 & 11.3 & 16.5 & 7.0 & 13.7 & 10.1 & 13.6 \\
\hline Right medio-lat reaction shear (MN.s) & 0.6 & 0.3 & 0.4 & 0.2 & 0.5 & 0.2 & 0.5 & 0.3 \\
\hline Left medio-lat reaction shear (MN.s) & 0.7 & 0.2 & 0.6 & 0.3 & 0.5 & 0.3 & 0.6 & 0.3 \\
\hline Extensor moment (MNm.s) & 2.1 & 0.6 & 2.2 & 0.7 & 1.9 & 0.3 & 2.1 & 0.5 \\
\hline Flexor moment (kNm.s) & 2.7 & 2.4 & 2.7 & 3.9 & 1.8 & 3.9 & 2.4 & 3.4 \\
\hline Right lateral bend moment (MNm.s) & 0.2 & 0.1 & 0.2 & 0.1 & 0.2 & 0.1 & 0.2 & 0.1 \\
\hline Left lateral bend moment (MNm.s) & 0.2 & 0.1 & 0.1 & 0.1 & 0.2 & 0.1 & 0.2 & 0.1 \\
\hline Right axial twist moment (kNm.s) & 33.4 & 15.5 & 22.6 & 7.6 & 24.0 & 6.3 & 26.6 & 11.5 \\
\hline Left axial twist moment (kNm.s) & 34.8 & 11.5 & 26.6 & 11.9 & 24.2 & 9.7 & 28.5 & 11.8 \\
\hline
\end{tabular}


Table 3 - Stepwise multivariate analyses for both wool handler skill level and LBP-Clin on cumulative percent time in working postures at $95 \% \mathrm{CL}$

\begin{tabular}{|c|c|c|c|c|c|c|}
\hline Cumulative \% time in: & Variable & Coeff. & Lower & Upper & Sig. & $\mathbf{R}^{2}$ \\
\hline \multirow[t]{2}{*}{ Mild Flexion } & Referent Group & 65.7 & 63.0 & 68.5 & & \\
\hline & Senior class & -5.4 & -10.17 & -0.58 & 0.029 & 0.08 \\
\hline \multirow[t]{2}{*}{ Neutral lateral bend } & Referent Group & 81.61 & 79.67 & 83.55 & & \\
\hline & Open class & -5.10 & -8.47 & -1.74 & 0.004 & 0.14 \\
\hline \multirow[t]{2}{*}{ Mild lateral bend } & Referent Group & 15.86 & 14.30 & 17.41 & & \\
\hline & Open class & 3.97 & 1.28 & 6.66 & 0.005 & 0.13 \\
\hline \multirow[t]{2}{*}{ Neutral axial twist } & Referent Group & 69.81 & 64.72 & 74.91 & & \\
\hline & Senior class & 10.94 & 2.12 & 19.76 & 0.016 & 0.10 \\
\hline \multirow[t]{2}{*}{ Mild axial twist } & Referent Group & 28.96 & 24.00 & 33.92 & & \\
\hline & Senior class & -10.21 & -18.81 & -1.62 & 0.021 & 0.09 \\
\hline \multirow[t]{2}{*}{ Severe axial twist } & Referent Group & 0.78 & 0.53 & 0.99 & & \\
\hline & Open class x LBP & 1.49 & 0.90 & 2.09 & $<0.0001$ & 0.31 \\
\hline \multicolumn{7}{|c|}{$\begin{array}{l}\text { Note } \\
\text { a) all coefficients for each response variable come from the final model. } \\
\text { b) the explanatory variables are listed in order of stepwise selection } \\
\text { c) the } R^{2} \text { values refer to the stepwise sequence of models. }\end{array}$} \\
\hline
\end{tabular}


Table 4 - Stepwise multivariate analyses for both wool handler skill level and LBP-Clin on cumulative loads at 95\% CL

\begin{tabular}{|c|c|c|c|c|c|c|}
\hline Cumulative load: & Variable & Coeff. & Lower & Upper & Sig. & $\mathbf{R}^{2}$ \\
\hline \multirow[t]{2}{*}{ Right medio-lateral reaction shear (kN.s) } & Referent Group & 0.42 & 0.35 & 0.50 & & \\
\hline & Open class & 0.20 & 0.07 & 0.33 & 0.004 & 0.14 \\
\hline \multirow[t]{2}{*}{ Left medio-lateral reaction shear (kN.s) } & Referent Group & 0.55 & 0.47 & 0.63 & & \\
\hline & Open class & 0.15 & 0.01 & 0.29 & 0.033 & 0.08 \\
\hline \multirow[t]{2}{*}{ Right lateral bend moment (kNm.s) } & Referent Group & 0.18 & 0.16 & 0.21 & & \\
\hline & Open class & 0.06 & 0.01 & 0.10 & 0.012 & 0.10 \\
\hline \multirow[t]{2}{*}{ Left lateral bend moment (kNm.s) } & Referent Group & 0.14 & 0.12 & 0.17 & & \\
\hline & Open class & 0.07 & 0.03 & 0.12 & 0.002 & 0.15 \\
\hline \multirow[t]{2}{*}{ Left axial twist moment (kNm.s) } & Referent Group & 23.28 & 19.94 & 26.63 & & \\
\hline & Open class & 10.09 & 4.30 & 15.89 & 0.001 & 0.17 \\
\hline \multirow[t]{2}{*}{ Right axial twist moment (kNm.s) } & Referent Group & 25.40 & 21.91 & 28.89 & & \\
\hline & Open class & 9.36 & 3.32 & 15.40 & 0.003 & 0.14 \\
\hline \multicolumn{7}{|c|}{$\begin{array}{l}\text { Note } \\
\text { a) all coefficients for each response variable come from the final model. } \\
\text { b) the explanatory variables are listed in order of stepwise selection } \\
\text { c) the } R^{2} \text { values refer to the stepwise sequence of models. }\end{array}$} \\
\hline
\end{tabular}




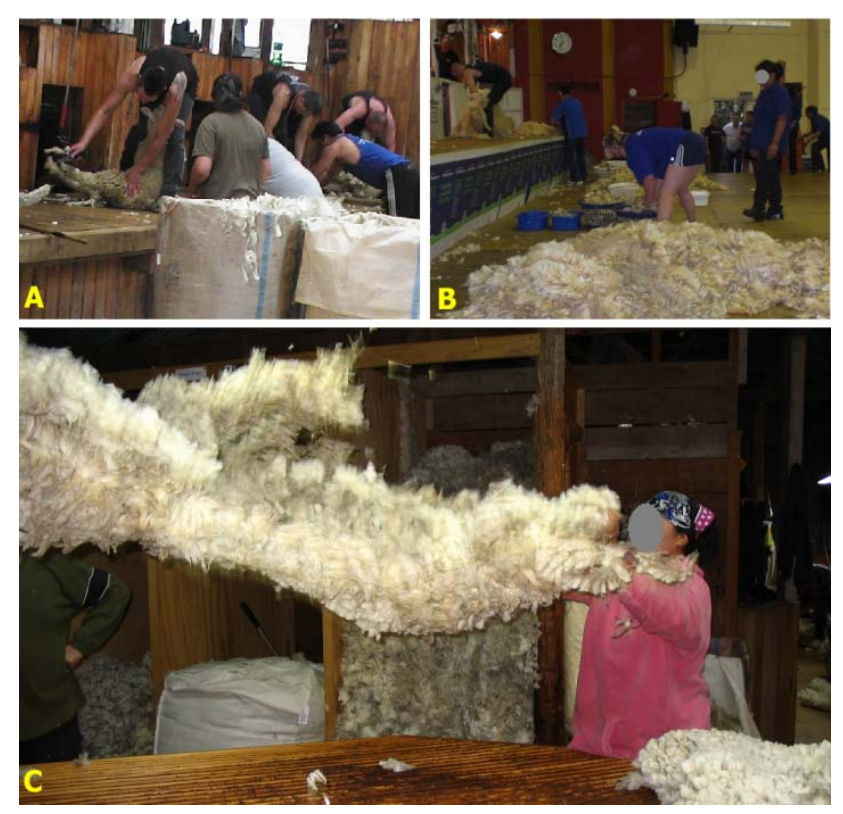

